\title{
Dentists run 423 miles for mental health charity
}

Three Scottish dentists have completed a gruelling 423-mile run covering the entire length of Scotland to support SAMH: the Scottish Association for Mental Health.

Stuart Campbell, 43, a specialist

prosthodontist and Ryan Stewart, 30, a GDP - both of Loanhead Dental Practice, Midlothian - were joined in completing the 'Running Scotland' challenge by Paddy Watson, 36, a dental researcher at Glasgow Dental Hospital. A fourth dental runner, Derek Marner, a GDP from Greenock, also started the challenge. Unfortunately for Derek, injury led to his withdrawal, but not before he completed 200 miles of the ultra run.

The route began in John O'Groats on 28 September with the exhausted dental trio arriving at the English border at Gretna Green 11 days later.

The dentists - all long-distance runners - were inspired to devise and then take up the challenge during lockdown when the pandemic led to the temporary closure of dental practices and cancelled most organised races.

They commented: 'We wanted to do something a bit different, to reconnect with our country and to highlight the huge positive benefits that running can have on mental wellbeing especially during challenging times'

Via a tracking device, friends, family and colleagues were able to track the 423-mile journey, which involved running around 40 miles a day, for eight hours a day, over 11 days.

Along the way, the dentists were joined by other dental runners of all abilities and were invited to stop off in dental practices for tea and cakes along the route.

Stuart Campbell said that it was 'the hardest challenge any of us have ever had to do, battling the often brutal rain and wind and running on broken feet and in pain day after day. It was at times extremely difficult but we were overwhelmed by the constant messages of support we received from the

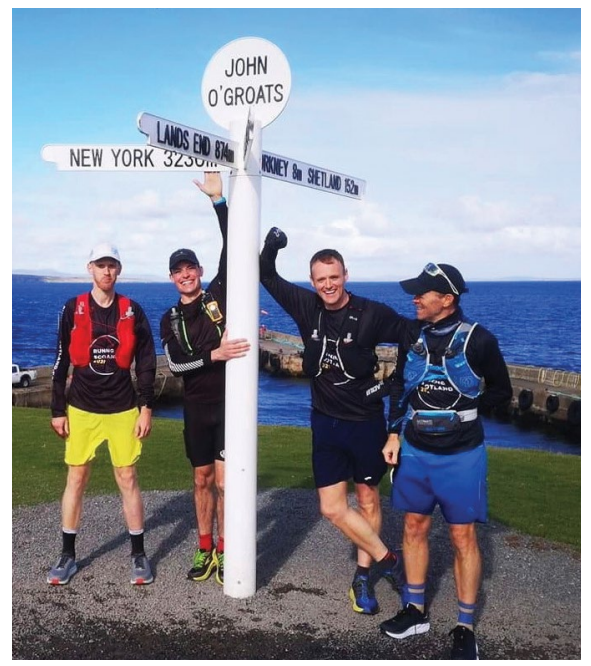

The start line at John O'Groats. Left to right: Paddy Watson, Ryan Stewart, Derek Marner, Stuart Campbell

dental community. The generosity of our supporters, raising so much money for SAMH and getting over the finishing line made it all worth it'.

To date, Running Scotland has raised $£ 11,500$ for $\mathrm{SAMH}$ 